\title{
Unusual Clinical Presentations of Malignant Melanoma: A Review of Clinical and Histologic Features with Special Emphasis on Dermatoscopic Findings
}

\author{
Raúl Cabrera ${ }^{1}$. Francisca Recule ${ }^{1}$
}

Published online: 30 October 2018

(c) The Author(s) 2018

\begin{abstract}
This review presents the main challenges encountered when diagnosing unusual variants of malignant melanoma with the aim of raising awareness to allow application of the most appropriate treatment strategies. Although these melanomas are often rare, their misdiagnosis potentially jeopardizes patients' health and survival, and has medicolegal implications. The clinical and histologic presentations of melanoma vary greatly, and assessment of uncommon melanomas can be difficult for practitioners because of their scarcity and resemblance to other dermatologic entities. The most problematic melanoma types are desmoplastic melanoma, polypoid melanoma, primary dermal melanoma, verrucous malignant melanoma, pigmented epithelioid melanocytoma, mucosal melanoma, follicular melanoma and melanoma with non-melanocytic differentiation. The two most difficult-to-diagnose subtypes of melanoma are the nevoid and the amelanotic melanomas. Some specific attributes of these variants can be more easily recognized with digital dermatoscopy, facilitating early detection and possibly avoiding invasive procedures. Key cases with the most notable clinical, dermatoscopic, and histopathologic features are presented, highlighting the practical issues of making an accurate diagnosis and choosing the best therapy.
\end{abstract}

\section{Key points}

Rare variants of malignant melanomas, usually with a poor prognosis, are often misdiagnosed or diagnosed late and are thereby potentially life threatening

Description of the morphologic and dermatoscopic features of these unusual variants of malignant melanoma will help practitioners to better identify them and adapt their management

\section{Introduction}

Malignant melanoma (MM) is one of the most challenging cancers to diagnose because it requires high levels of expertise from the dermatologist to detect the lesions at an early

Raúl Cabrera

rcabrera@alemana.cl

Department of Dermatology, Universidad del Desarrollo, Clínica Alemana, Manquehue Norte 1410, Vitacura,

Santiago, Chile stage and from the histopathologist to interpret the complex architecture of the skin biopsies [1]. Moreover, MMs exhibit various clinicopathologic and cytologic manifestations. Recognition of some MM variants with unusual presentations is even more difficult because of their scarcity.

Although uncommon MM variants generally account for less than $2 \%$ of all melanomas [2-6], their prognosis is often poor, and their misdiagnosis may lead to potentially harmful handling errors associated with medical malpractice claims [7-9]. Early diagnosis of MMs is thus of vital importance for appropriate management and a successful outcome.

In many instances, dermatoscopy may help to determine the malignant potential of the lesion and to discriminate between the clinicopathologic variants of MM [10]. This article provides an overview of the clinical, dermatoscopic, and histologic characteristics of the MM subtypes with the highest risk of diagnostic failure, with the aim of helping practitioners to improve the differential diagnosis of these rare MMs and reduce the risk of harmful consequences on patient survival. 


\section{Desmoplastic Melanoma}

Desmoplastic melanoma (DM) accounts for less than $4 \%$ of all MMs [11]. It is twice more common in men and occurs mostly in individuals $\geq 60$ years of age. [12] It is mainly found in sun-exposed areas of the skin, especially the head and neck $(51 \%,[12,13])$. It can arise de novo or together with other MM types, such as lentigo maligna melanoma (LMM) or other in-situ melanomas. Two histologic subtypes of DM have been defined, depending on the degree of cellularity and/or desmoplasia: pure DM and mixed DM. The clinical presentation of DM is often non-specific with lesions showing local growth, a palpable nodule or plaque, and an absence of pigmentation in about $60 \%$ of cases [14], especially in the pure type (with prominent fibrosis). These lesions can therefore be mistaken clinically for a scar, a fibroma, basal cell and squamous cell carcinomas, or fibromatosis, delaying their correct diagnosis and treatment. As a result of their association with DM, Chen et al. recommend palpation of all LMM to confirm the absence of a nodular lesion, which may be a DM component [13]. Mixed DM, with features of desmoplastic and non-desmoplastic melanoma, may be easier to identify as clinical pigmentation is more frequent in this subtype and it is more often associated with LMM or superficial spreading melanoma than pure DM [13].
Under dermatoscopy, the only diagnostic clues for hypopigmented or amelanotic lesions are the shiny white scar-like structureless areas and atypical vascular patterns, such as dotted or linear-irregular vessels (also known as serpentine vessels) and milky-red areas (Fig. 1, panels a-1 to b-4) [13, 15]. In two studies analyzing DM cases, all lesions had at least one melanoma-specific structure and regression structures visible as peppering [13]. Sometimes, DMs have little gray dots, atypical vessels, atypical globules, or a negative pigmented network. Dermatoscopy is particularly useful on pigmented lesions owing to the presence of an atypical network, a pseudonetwork, or rhomboidal structures [16].

At diagnosis, which is often late, most DMs are thick with a mean Breslow depth of between 2.0 and $6.5 \mathrm{~mm}$, significantly greater than that found with non-DMs [13].

Nevertheless, at similar depths of invasion, DMs are associated with a lower risk of metastases than other melanomas. In addition, pure-type DMs have a less aggressive clinical course than mixed DMs, with a 5-year melanoma-specific mortality rate of 11 vs. $31 \%(p<0.01$ [17]). Therefore, a distinction between these subtypes of DM is important for therapeutic decision making. The transcription factor SOX10 expressed in more than $95 \%$ of cases can also be used as a sensitive marker for DM diagnosis $[18,19]$.
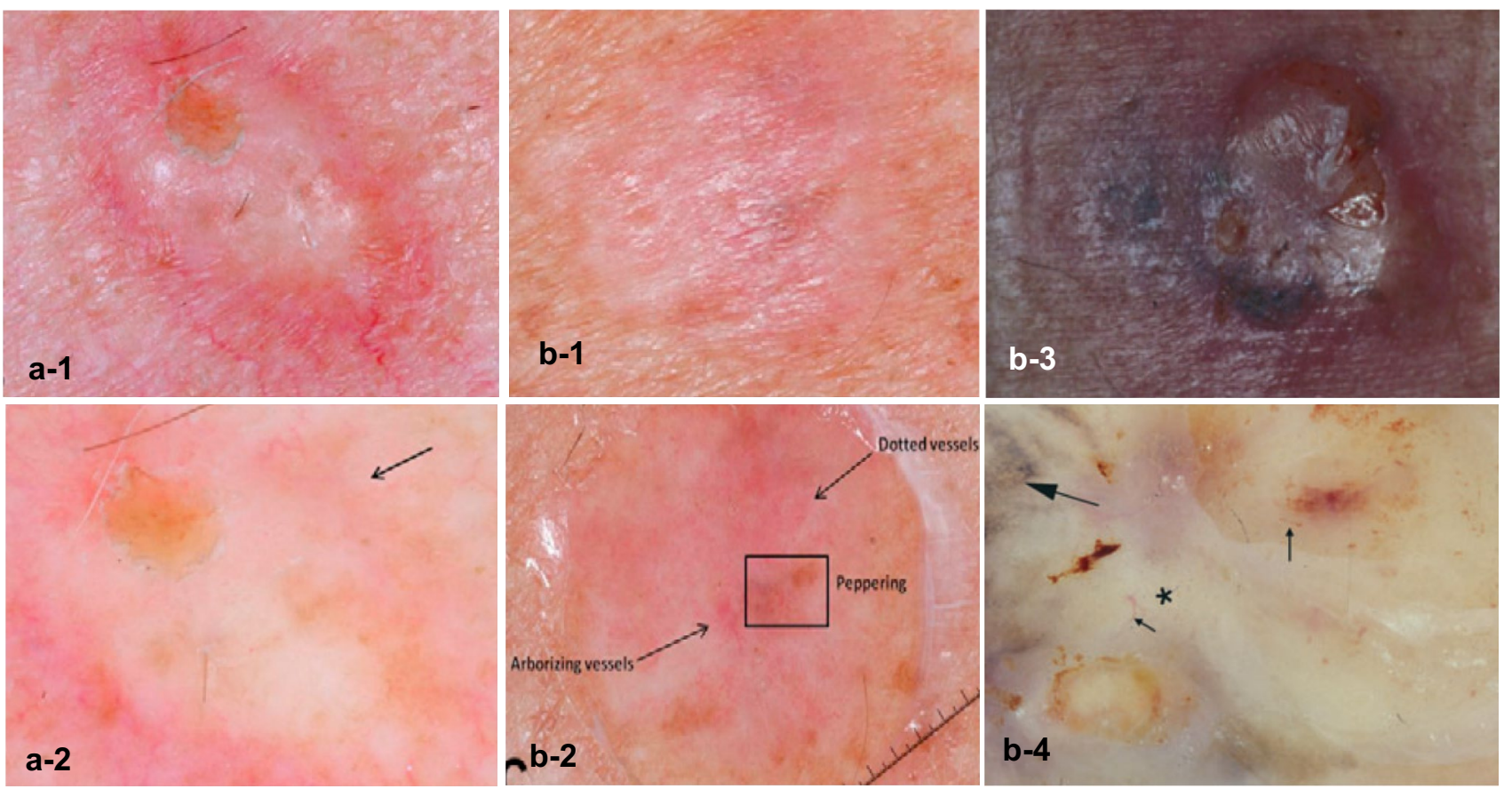

Fig. 1 Images of desmoplastic melanomas (a-1 to b-2 from Chen et al. [13] and b-3 to b-4 from Debarbieux et al. [15]). a Pure type: a-1 close-up photograph of a firm cystic to scar-like nodule and a-2 dermatoscopy showing atypical dotted vessels (arrow). b Mixed type: b-1, b-3 close-up photograph, and b-2, b-4 dermatoscopy shows regression structures (box in b-2), dotted and arborizing vessels (arrows in b-2), a regression pattern 'peppering' (big arrow in b-4), linear irregular vessels (small arrows in $\mathbf{b - 4}$ ), and a white scar-like structureless area (asterisk in b-4) 


\section{Polypoid Melanoma}

Although a polypoid configuration may sometimes be encountered in other melanoma types [20], polypoid melanoma is commonly considered as a clinical variant of the nodular form of melanoma in which the volume of the tumor is located above the skin surface (in at least $50 \%$ of cases). This type of MM may be sessile or attached to the skin with a peduncle. It is characterized by exophytic growth and frequent ulceration. It is not necessarily pigmented, which may cause it to be mistaken for benign lesions such as pyogenic granuloma, hemangioma, intradermal nevus, polypoid Spitz nevus, or fibroepithelial polyp [21]. It is not only found on the skin but also in mucosae, including the esophagus, upper respiratory tract, rectum, vagina, and biliary tract [6]. Cutaneous polypoid melanoma shows rapid growth with active vascularization, which explains its aggressiveness and the appearance of metastases within a few weeks (Fig. 2, panel a) [22]. No genetic data are currently available for this type of melanoma.

Polypoid melanoma mainly affects young people (20-39 years of age, [22]) and has a poor prognosis owing to a high incidence of ulceration and deep penetration reflected by a high Breslow index. As a result, the 5-year survival rate for this type of melanoma is low, ranging from 32 to $42 \%$, as compared with $57 \%$ for the nodular melanoma subtype and $77 \%$ for the superficial melanoma subtype [22].

\section{Primary Dermal Melanoma}

Primary dermal melanoma (PDM) is a very rare subtype of melanoma $(0.8 \%$ of all MMs [2, 23]), exclusively confined to the dermis. In a case series of 49 patients, the mean age was 51.2 years and the most common sites involved were the trunk, the head and neck, the extremities, and the acral areas [23]. Morphologically, PDM is almost indistinguishable from cutaneous metastatic melanoma (CMM), but it has a lower metastatic potential and a much better prognosis with a long-term survival rate of $80-100 \%$, compared with $24 \%$ for cutaneous metastatic melanoma stage IIIC, although deep penetration can occur (mean Breslow depth of $3.01 \mathrm{~mm}$ and Clark level $\geq$ III) [23]. The following classification of PDM according to four distinct morphologic patterns has been proposed: Spitzoid type, blue nevus-like, PDM with an associated intradermal nevus, and a "not otherwise specified" group. Moreover, association with a nevus component, lack of subcutaneous involvement, and a younger age at presentation were found to be hallmarks of PDM [23]. None of the lesions described have shown evidence of regression.

Digital dermatoscopy was used for the first time for PDM in a case report documenting a lesion with several papules with a pigmented "vascular-like" aspect (Fig. 2, panel b)
[24]. Findings of "papillomatous projections" with blotchy light brown to pink and dark brown areas with the addition of a blue hue led to suspicion of a pigmented lesion that was later confirmed as an intradermal primary melanoma. Conversely, the main phenotypic features of MM with epidermal infiltration, such as an atypical pigmented network and irregular streaks, were not found. Hence, the dermoscopic profile of an equivocal lesion is useful to help guide further molecular testing when PDM is suspected.

Immunohistochemistry, fluorescence in-situ hybridization, and gene expression profiling are other useful methods for further characterizing these tumors and distinguishing them from CMMs [23, 24]. Given that most conventional staging parameters used for prognosis in cutaneous melanoma have limited applicability in PDM, combining these complementary approaches allows better differentiation of PDMs from CMMs, and allows the proposal of adapted treatments.

\section{Verrucous Malignant Melanoma}

Verrucous MM accounts for around 3\% of melanomas [25] and clinically resembles pigmented verrucous nevi or seborrheic keratosis, a prevalent benign epidermal tumor affecting the sun-exposed areas of the skin. Therefore, misdiagnosis of verrucous MM is common, occurring in over $50 \%$ of cases, [25] with subsequent implications for therapeutic management and patient prognosis.

This variant can occur de novo or can develop with any of the following histopathologic types: superficial spreading melanoma, LMM, nodular melanoma, or acral lentiginous melanoma. These tumors are most often located on the head and the limbs. All subtypes affect women and men with an equal frequency and have a similar prognosis with cure rates of between 95 and $100 \%$ [25].

Dermatoscopic inspection of verrucous MM enables the detection of specific patterns suggestive of melanocytic lesions, such as dots, globules, streaks, and a bluish-white veil (Fig. 2, panel c). Peppering can also appear in some areas. Nevertheless, the presence of comedo-like openings and milia-like cysts might lead to confusion as they are also commonly found in seborrheic keratosis [26].

Lesions suspected of being malignant on dermatoscopy or those for which the nature cannot be clearly established, must be surgically excised with a narrow border [27]. Confirmation of the diagnosis with a complete histopathologic inspection is warranted to allow the appropriate final treatment procedure to be applied according to conventional guidelines [28]. 

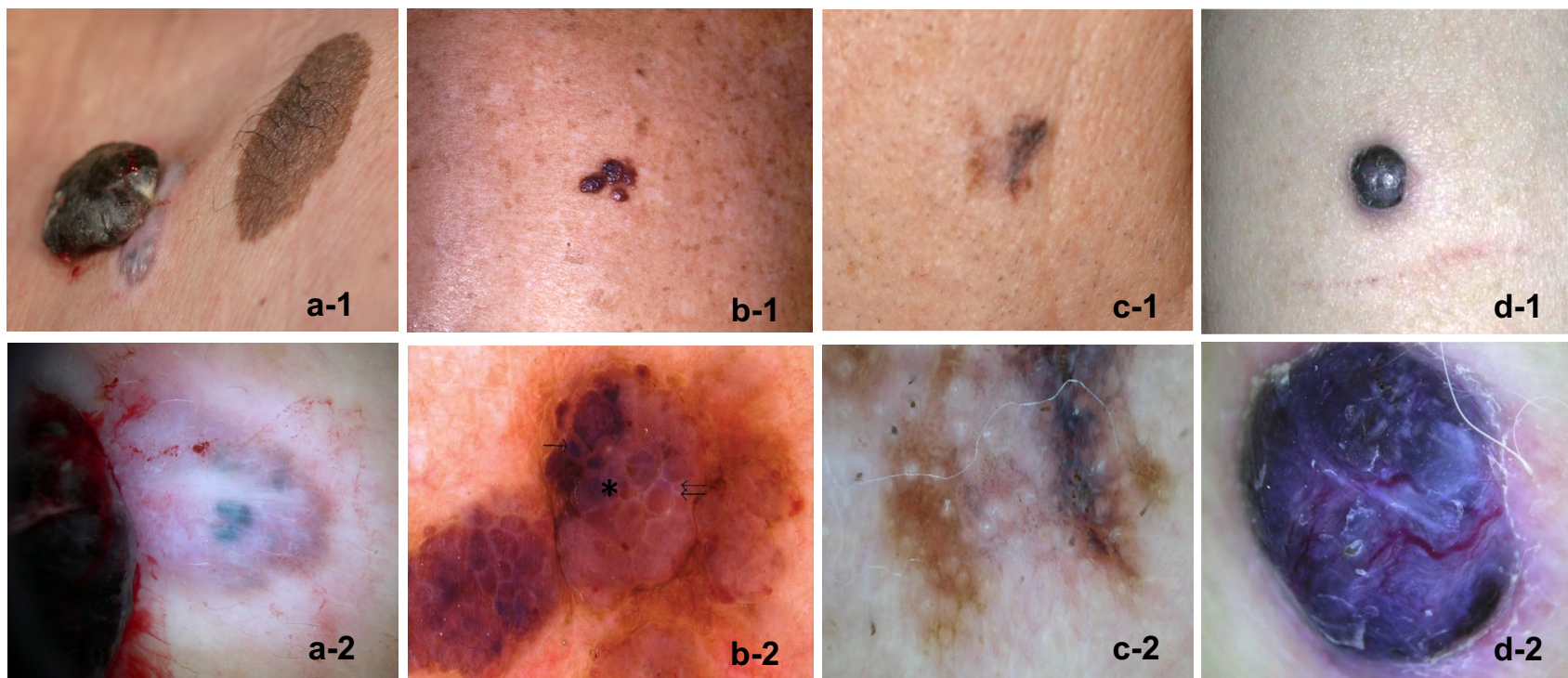

Fig. 2 Images of four unusual subtypes of malignant melanoma. a Polypoid melanoma (Cabrera R, personal images). a-1 Close-up photograph of a $3-\mathrm{cm}$ diameter, pedunculated pigmented lesion on the posterior trunk, in which the upper part is attached to the brown-blue pigmented skin with a colorless peduncle of only $0.5 \mathrm{~cm}$. a-2 Dermatoscopy shows a blue-white veil in the exophytic lesion, and big bluegray nests and whitish areas in the peduncle's base. b Primary dermal melanoma (from Cabrera et al. [24]). b-1 Close-up photograph of a pigmented vascular lesion on the thorax. b-2 Dermatoscopy shows dark brown blotchy areas in some papillomatous projections (sin- gle arrow), areas with a light brown-pink color (double arrow), and a 'blue-hue' color without a veil (asterisk). c Verrucous malignant melanoma (Cabrera R, personal images). c-1 Close-up photograph of a verrucous lesion on the face. c-2 Dermatoscopy shows bluish-white veils with dots and globules, and peppering in some areas. d Pigmented epithelioid melanocytoma (from Aviles-Izquierda et al. [30]). d-1 Close-up photograph of a pigmented nodule on the arm. d-2 Dermatoscopy shows a homogeneous blue lesion with whitish structures, a blue-white veil, and polymorphic vessels

\section{Pigmented Epithelioid Melanocytoma}

This type of melanoma was first described in horses, which led to the same entity in humans being referred to as "animal-type melanoma" [1]. The term pigmented epithelioid melanocytoma has now been adopted. Pigmented epithelioid melanocytoma is a dermal melanocytic proliferation with heavily pigmented epithelioid and spindled melanocytes. Although it is not well described, it seems to display clinical, histologic, and prognostic characteristics that differ from other melanoma subtypes.

Pigmented epithelioid melanocytoma appears to occur with equal frequency in men and women, most often in the extremities $(38.3 \%)$, the head and neck $(30.8 \%)$, and the trunk (30.8\%) [29]. At diagnosis, the median Breslow depth of the lesions was $3.8 \mathrm{~mm}$, and ulceration was reported in $15.8 \%$ of cases. The presence of a lesion since birth or childhood (34.4\% of cases) that expanded in later years has been reported as a distinct clinical feature of this melanoma [29].

These lesions can present as nodules or plaques and show all the dermoscopic features of MM with a blue-black or blue appearance, and a blue-white veil or irregular white areas (Fig. 2, panel d) [30]. As for other forms of MM, immunohistochemical analysis usually shows positivity of pigmented epithelioid melanocytoma for S-100 protein, vimentin, HMB-45, and Melan-A [29]. Further molecular characterization is necessary to differentiate this rare melanoma from malignant epithelioid blue nevus, deep penetrating nevus, and metastases of PDM.

\section{Mucosal Melanoma}

The mucosal melanoma is an extremely rare type of melanoma (around 1\% of MMs, personal data, and [3]) that mainly occurs in the head and neck (25-50\% of cases [31]). Primary tumor sites are most often located in the nasal cavity (49.1\%), paranasal sinuses (23.1\%), and oral cavity (18.8\%). Lesions can occur more rarely in the nasopharynx (5.5\%), the parotid gland (2.9\%), and to a lesser extent, in the anorectal and vulvovaginal regions and the urinary tract [32].

The major pitfalls for the diagnosis of primary mucosal melanoma are its hidden location and the absence of early symptoms, often leading to a late diagnosis of already advanced tumors with a poor prognosis (average 5-year 

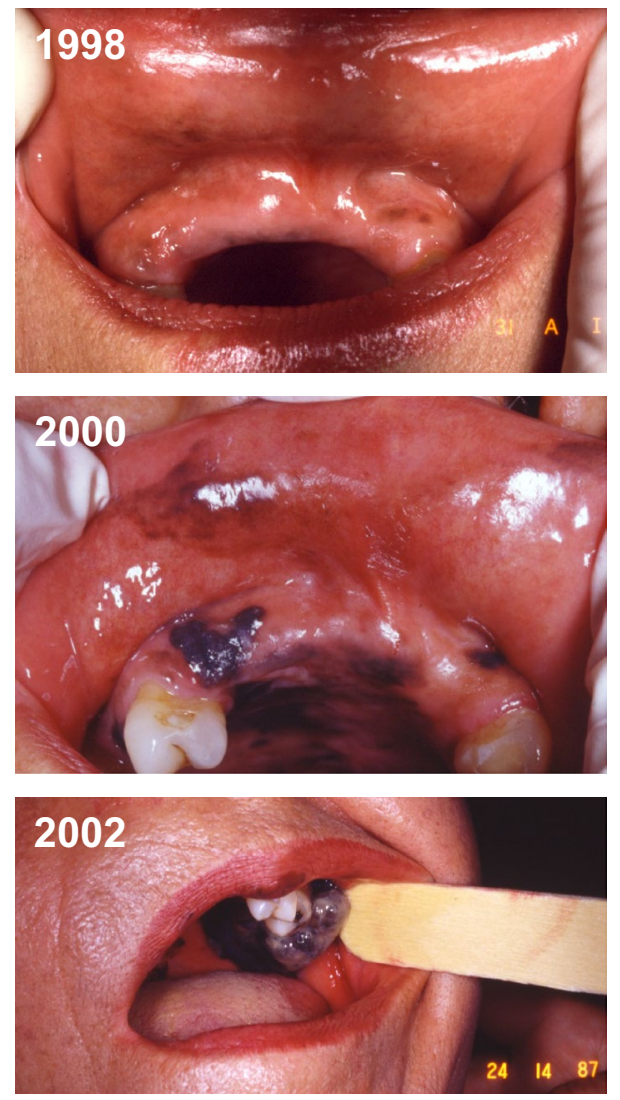

Fig. 3 Case report of a mucosal melanoma (Cabrera R, personal images). Progression over 4 years of a mucosal melanoma in the oral cavity of a 70-year-old woman. Pigmentation was present initially and the lesion changed rapidly. The patient initially refused the biopsy (1998) but 2 years later an examination confirmed it was a melanoma (2000). However, the patient decided not to be treated. In 2002, surgery was finally performed, and the sentinel lymph nodes appeared to be totally pigmented (not shown)

overall survival rate $<30 \%$ ) [33]. Previous pigmentation at the site of the lesion was reported in some patients, but usually these lesions were not initially considered as a malignant condition. However, they can rapidly change and may have a very poor outcome with rapid invasion of lymph nodes (Fig. 3). Breslow depth alone seems to be useless in the staging of the majority of primary mucosal melanomas. Interestingly, overexpression of the c-KIT tyrosine kinase, a key regulator of melanocytes, has been described in a great number of mucosal melanoma cases (from 39 to $88 \%$ depending on the study) and could be a marker or even a target for KIT inhibitors for the treatment of metastatic mucosal melanomas $[3,31]$.

\section{Nevoid Melanoma}

Nevoid melanoma (NeM) is deemed to be one of the most difficult melanomas to diagnose as it mimics a benign nevus but behaves like an invasive melanoma, with local recurrences and metastases [1]. Typically, it presents as a black or brown nodule (59\% of cases), which can be dome shaped or verrucous, arising on the trunk or limbs of adult patients [34]. Lesions can also have a plaque-type topology (30\%) or be papule-like (11\%). The histopathology of this tumor often lacks the conventional attributes of melanoma, i.e., asymmetry, poor circumscription, or pagetoid growth of melanocytes [6], and is not typified by ulceration [5]. It is worth noting that no in-situ NeM exists. Although NeM accounts for less than $1 \%$ of all melanomas, it is associated with a high rate of mortality (15-37.5\%) [5].

Nevoid melanoma can be classified into three main groups according to the most frequently associated dermatoscopic features [34]: nevus-like NeM (48\%), amelanotic $\mathrm{NeM}(30 \%)$, and tumors with a multi-component pattern $(15 \%)$, while a few tumors with a mixed pattern $(7 \%)$ remain unclassified. Nevus-like NeM display a papillomatous architecture with atypical vessels, irregular dots/globules, and/ or multiple milia-like cysts [34]. They can either present an irregular pigmentation or have a homogenous color pattern from bluish to reddish brown. Amelanotic NeMs are characterized by an atypical vascular pattern with linear irregular vessels or serpentine, dotted and/or glomerular vessels [34]. They can sometimes manifest multiple milia-like cysts that are often associated with benign lesions. The third type of NeM shows the key criteria of superficial spreading melanoma, including irregular pigmentation and shape, and a blue-white veil, which allows prompt diagnosis ([34] and Fig. 4, Cabrera R, personal images). In all NeM types, suspicion of a melanoma is therefore supported by the presence of an atypical vessel pattern and irregular dots/globules, and by a lack of comma vessels, which are commonly seen in intradermal nevi. Moreover, in a sample of 27 lesions, the mean Breslow thickness was $3.2 \mathrm{~mm}$ [34].

\section{Amelanotic Melanoma}

Alongside NeM, the most problematic melanoma tumors to identify are hypomelanotic/amelanotic melanomas. Clinicians do not suspect melanoma in the first instance when lesions are poorly or not pigmented and devoid of specific traits of melanoma. They can be mistaken for benign 
melanocytic or non-melanocytic neoplasms, or for dermatitis, often leading to a late diagnosis and delayed treatment. The prognosis for patients with amelanotic metastases is worse than that for patients with pigmented metastases [35]. Thus, misdiagnosis of amelanotic melanoma remains a significant cause of diagnostic error. As a result, this lesion has been called "the great masquerader" [36, 37].

This type of lesion is most often located on the trunk, the head and neck, and in the lower part of the limbs [4, 35]. The three morphologies found in a series of 75 cases were epithelioid (72\%), spindled (18.7\%), and desmoplastic (5.3\%) [4]. Dermatoscopy combined with immunohistochemical staining can assist clinicians in the diagnosis of such skin lesions. Several dermatoscopic features appear to be associated with amelanotic melanomas, in particular, vascular structures [35, 38]: polymorphic arrangement of vessels, a blue-white veil, multiple blue-gray dots, milky red-pink areas, an asymmetric shape, multiple colors, ulceration, and a scar-like depigmentation, but more specific morphology is the association of dotted vessels with other types of vessels (linear irregular, looped, or serpentine vessels). Conversely, some dermatoscopic patterns argue against amelanotic melanoma: a symmetric pigmentation pattern, irregular and multiple blue-gray globules, multiple milia-like cysts, and predominantly comma vessels [38].

Hypomelanotic melanoma is also often difficult to diagnose, as exemplified in a patient with an extensive chronic foot ulcer, in whom attentive dermatoscopic observation revealed that the only atypical pigmented area was at the periphery (Fig. 5). The excised lesion was found to be a focally pigmented subcutaneous lesion in the deep dermis and hypodermis, confirming the diagnosis of a hypopigmented melanoma.

Specific antibodies may aid in detecting malignant cells on the biopsy, such as immunostaining of MITF (microphthalmia-associated transcription factor) [4]. Little is known about the genetic and molecular characteristics of this peculiar type of MM. Classically, BRAF mutations have been associated with highly pigmented MMs. However, Massi et al. demonstrated a high BRAF mutation rate in amelanotic melanoma, suggesting that this characteristic could be used as a valuable diagnostic tool [39].

\section{Follicular Malignant Melanoma}

Follicular MM (FMM) is a rare variant of MM that originates in most cases on the photodamaged skin of elderly patients. The follicular origin of the neoplastic cells is still a matter of debate. It remains to be determined whether FMM is a primary MM of the melanocytes in the hair follicle or whether it represents follicular colonization of intraepidermal neoplastic melanocytes [40]. Tjarks et al.

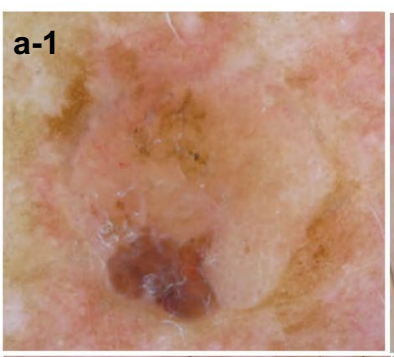

a-2
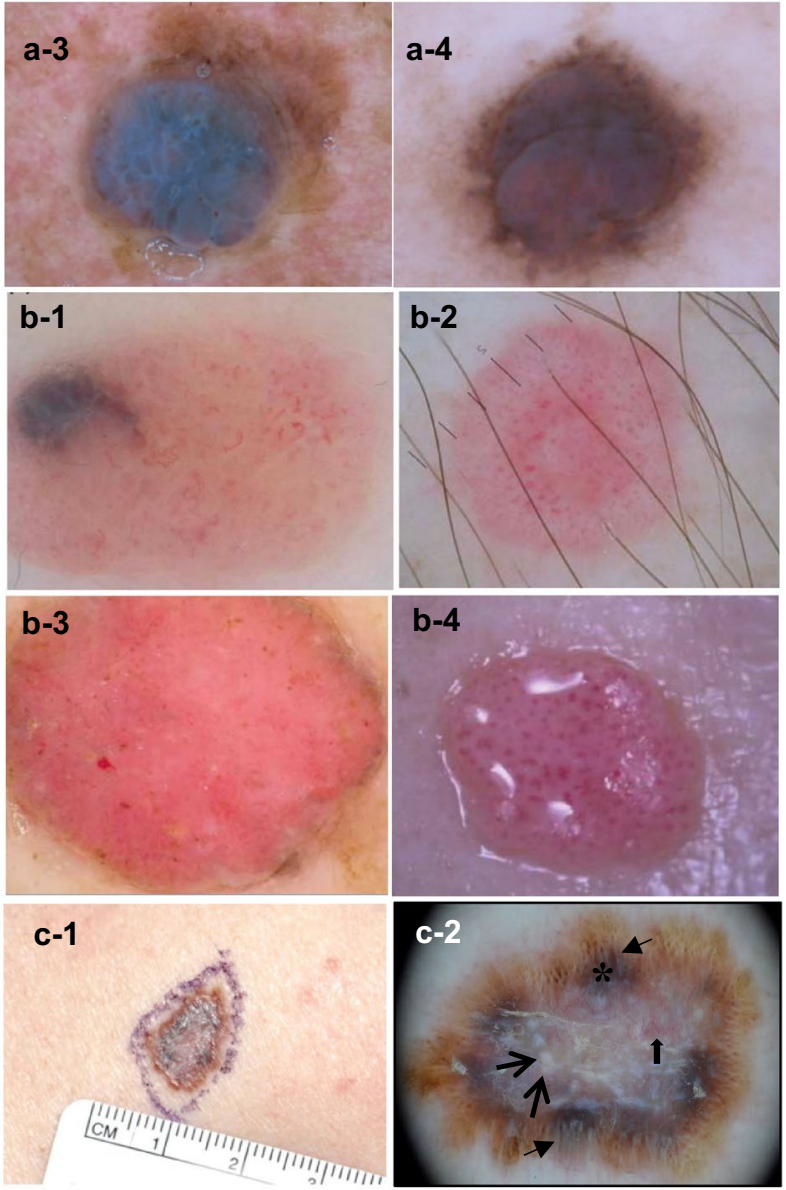

Fig. 4 Dermoscopic images of the three main groups of nevoid melanoma (a-1 to b-4 from Longo et al. [34]). a Nevus-like nevoid melanomas. All lesions show a nevus-like appearance with colors varying from skin-colored (a-1) to light brown (a-2), bluish (a-3) and reddish brown (a-4). Irregular pigmentation (a-1, a-3) and irregularly distributed globules (a-2, a-4) are observed. b Amelanotic nevoid melanomas. All tumors have an atypical vascular pattern, with either linearirregular vessels or serpentine (b-1), dotted $(\mathbf{b}-\mathbf{2}, \mathbf{b}-\mathbf{3})$ or glomerular vessels (b-4). Multiple milia-like cysts can be seen (b-3). c Nevoid melanoma with a multicomponent pattern (Raúl Cabrera personal images). A delimited plaque of $1.2 \times 1.5 \mathrm{~cm}$ shows a brown, black, slightly raised periphery and whitish center (c-1). Dermatoscop (c-2) shows linear projections at the periphery (black arrows) with brown dots and globules $(*)$, chrysalis in the center (blue arrows) and arborizing vessels (thick blue arrow)

recently defined a primary FMM as a melanoma that originates in the hair follicle with a minimum epidermal component (with a lateral epidermal tissue invasion less than the 

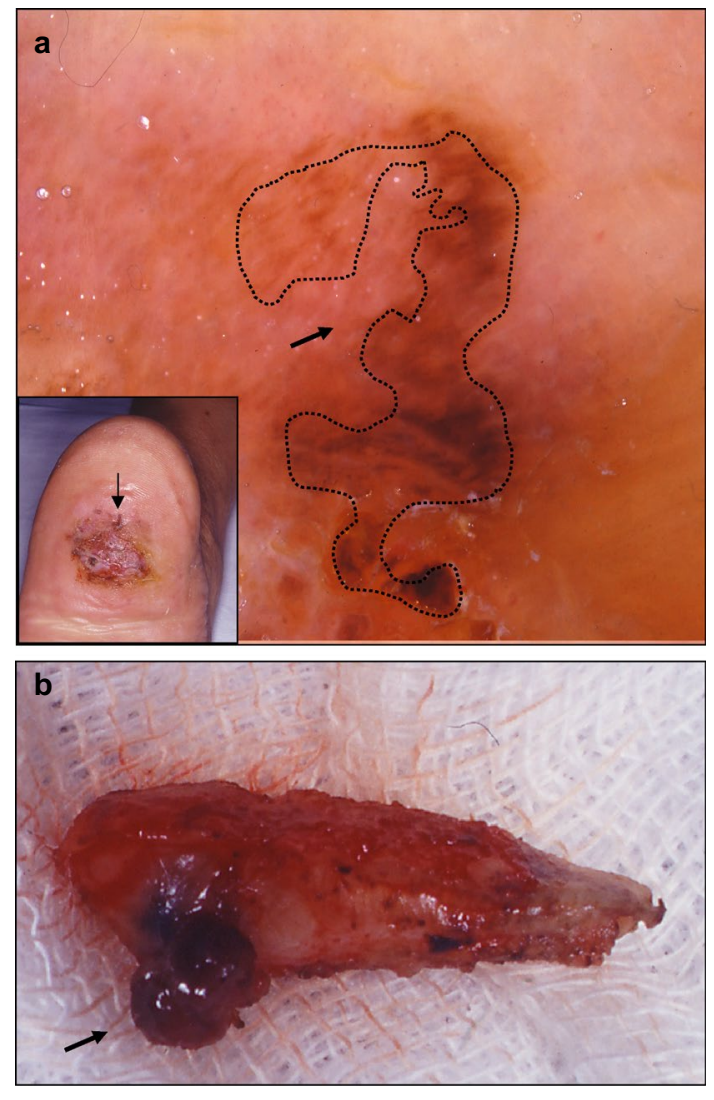

Fig. 5 Hypomelanotic melanoma (Cabrera R, personal images). a Chronic foot ulcer with a hypopigmented lesion. In the inset, the arrow shows the exclusively peripheral localization of the atypical pigmented area on a chronic foot ulcer. Dermatoscopy of this area shows an atypical red pigmentation on the periphery (arrow on the main image). b Biopsy of the lesion. The deep part of the dermis presents pigmentation (arrow)

depth of invasion around the hair follicle [41]). Follicular MM should be distinguished from folliculotropic primary MM and LMM with secondary follicular invasion (where the lateral epidermal compromise is more extensive than the depth). Another differential diagnosis is folliculotropic metastasis (secondary compromise of the hair follicle from another distant primary MM) [41]. Folliculotropic metastasis of cutaneous melanoma is rare, with only five published case reports [42], but a history of previous melanoma and the presence of multiple lesions favor its diagnosis [43, 44]. To our knowledge, only nine cases of FMM have been published to date, mainly in adults, but one case has been reported in a young patient with an absence of actinic elastosis [44].

Clinically, FMM presents as pigmented macules that are characteristically smaller than $0.5 \mathrm{~cm}$ in diameter, pigmented cystic lesions, or pseudocomedones, mainly in photo-exposed areas [40]. Hantschke et al. studied five

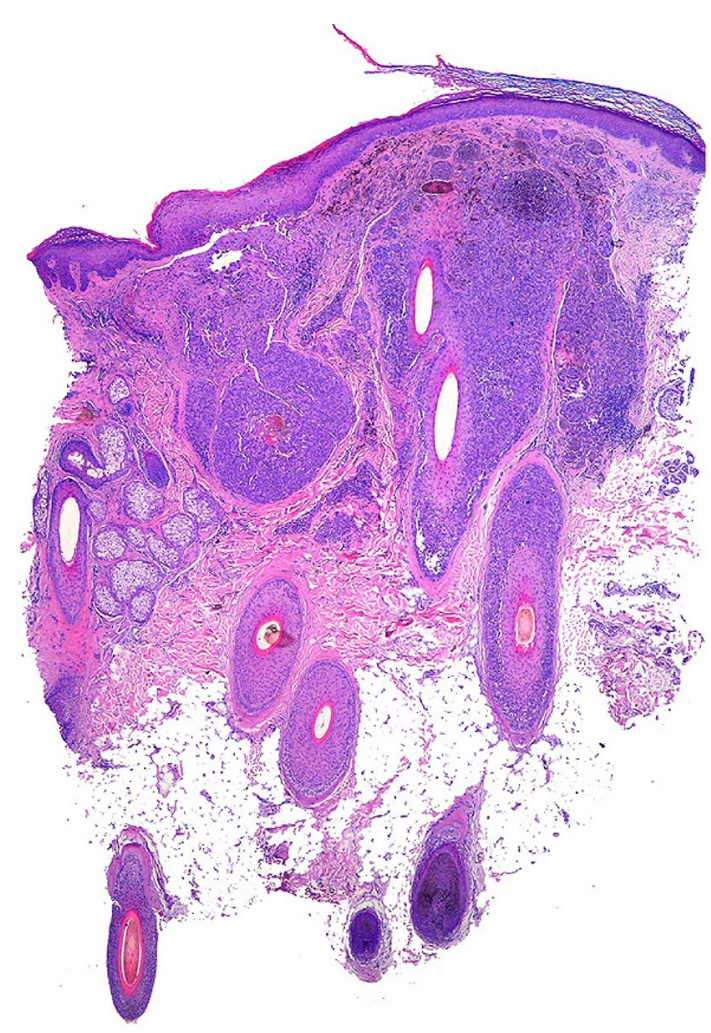

Fig. 6 Follicular melanoma (Cabrera R, personal images). Punch biopsy showing atypical melanocytes surrounding the hair shaft

cases of FMM, three located on the nose, one on the cheek, and one on the posterior cervical region [40]. Only one case of metastatic folliculotropic MM has been described using dermatoscopy, with a dark grayish blue homogeneous area surrounding a thick central hair and a weak grayish blue shadow in the upper part of the lesion, similar to the shadows observed in surrounding hairs [43].

Histopathology of FMM is characterized by compromise of one or two adjacent hair follicles in which the atypical melanocytic cells extend through the follicular epithelium and the sebaceous gland. However, secondary invasion of the surrounding epidermis and dermis has been described. The criteria for defining the degree of follicular invasion to make the diagnosis are still under investigation [45]. Hantschke et al. [40] and Tjarks et al. [41] proposed that the distance of the epidermal involvement on both sides of the hair follicle should not exceed the depth of the follicular structure, unlike for LMM in which the epidermal involvement is extensive. The atypical melanocytic cells can affect the hair follicle in its entirety or present a focal compromise [45], as in the lesion shown in Fig. 6. 


\section{Melanoma with Nonmelanocytic Differentiation}

Melanomas with non-melanocytic differentiation are even more unusual. Clinically, they are devoid of distinctive features, and their diagnosis is established only with the help of histopathology with specific tissue markers and classic MM immunochemistry. Some of these lesions have been described as non-specific macules (neural), reddish ulcerated nodules (smooth muscle tissue), or painful subungual nodules (cartilaginous tissue). Thus, histologically, MM very occasionally can present in non-melanocytic tissue or in cellular components, which is known as divergent differentiation or metaplastic change. Different types have been reported according to their differentiation: fibroblastic/myofibroblastic, neural, smooth muscle, ganglionic, rhabdomyoblastic, and neocartilage or chondroid differentiation. The latter tends to locate on acral areas, particularly the subungual region. Divergent differentiation is an exceptional phenomenon and diagnosis in these cases is particularly difficult [46-49].

\section{Conclusion}

The inability to recognize unusual MM variants is a real challenge for clinicians and pathologists and might have a critical impact on patients. Attentive examination and follow-up of patients, especially those at high risk, are required to improve early detection of rare MMs, ensuring that malignancies are not missed. Dermatoscopy can be used in the first instance to facilitate recognition of key features of some unusual MM subtypes. In addition, reflectance confocal microscopy has emerged as a valuable diagnostic device in MM, guiding the clinician in the decision-making process $[50,51]$. Furthermore, the use of histopathology, with specific immunohistochemical markers, as well as specific genetic tests can help in establishing a solid diagnosis of an unusual type of melanoma. After the tumor has been well characterized, the most appropriate therapeutic management can be applied, favoring a better clinical outcome.

Acknowledgements The authors thank Cécile Desjobert, Ph.D and Marielle Romet, Ph.D (Synergy Pharm) who provided medical writing assistance funded by Laboratoires dermatologiques Avène.

\section{Compliance with Ethical Standards}

Funding Medical writing assistance was funded by Laboratoires dermatologiques Avène.

Conflict of interest Dr R. Cabrera has received a speaker honorarium from Laboratoires dermatologiques Avène for the Entretiens d'Avène conference. Dr F. Reculé declares that she has no conflicts of interest that might be relevant to the contents of this manuscript.

Disclosure Statement This article is published as part of a journal supplement wholly funded by Laboratoires dermatologiques Avène.

Open Access This article is distributed under the terms of the Creative Commons Attribution-NonCommercial 4.0 International License (http://creativecommons.org/licenses/by-nc/4.0/), which permits any noncommercial use, distribution, and reproduction in any medium, provided you give appropriate credit to the original author(s) and the source, provide a link to the Creative Commons license, and indicate if changes were made.

\section{References}

1. Magro CM, Crowson AN, Mihm MC. Unusual variants of malignant melanoma. Mod Pathol. 2006;19(Suppl 2):S41-70.

2. Lee CC, Faries MB, Ye X, Morton DL. Solitary dermal melanoma: beginning or end of the metastatic process? Ann Surg Oncol. 2009; 16(3):578-84.

3. Mihajlovic M, Vlajkovic S, Jovanovic P, Stefanovic V. Primary mucosal melanomas: a comprehensive review. Int J Clin Exp Pathol. 2012;5(8):739-53.

4. Cheung WL, Patel RR, Leonard A, Firoz B, Meehan SA. Amelanotic melanoma: a detailed morphologic analysis with clinicopathologic correlation of 75 cases. J Cutan Pathol. 2012;39(1):33-9.

5. Zembowicz A, McCusker M, Chiarelli C, Dei Tos AP, Granter SR, Calonje E, et al. Morphological analysis of nevoid melanoma: a study of 20 cases with a review of the literature. Am J Dermatopathol. 2001;23(3):167-75.

6. Situm M, Buljan M, Kolic M, Vucic M. Melanoma-clinical, dermatoscopical, and histopathological morphological characteristics. Acta Dermatovenerol Croat. 2014;22(1):1-12.

7. Troxel DB. Medicolegal aspects of error in pathology. Arch Pathol Lab Med. 2006;130(5):617-9.

8. Moshell AN, Parikh PD, Oetgen WJ. Characteristics of medical professional liability claims against dermatologists: data from 2704 closed claims in a voluntary registry. J Am Acad Dermatol. 2012;66(1):78-85.

9. Rayess HM, Gupta A, Svider PF, Raza SN, Shkoukani M, Zuliani GF, et al. A critical analysis of melanoma malpractice litigation: should we biopsy everything? Laryngoscope. 2017;127(1):134-9.

10. Salerni G, Teran T, Puig S, Malvehy J, Zalaudek I, Argenziano G, et al. Meta-analysis of digital dermoscopy follow-up of melanocytic skin lesions: a study on behalf of the International Dermoscopy Society. J Eur Acad Dermatol Venereol. 2013;27(7):805-14.

11. Quinn MJ, Crotty KA, Thompson JF, Coates AS, O'Brien CJ, McCarthy WH. Desmoplastic and desmoplastic neurotropic melanoma: experience with 280 patients. Cancer. 1998;83(6):1128-35.

12. Feng Z, Wu X, Chen V, Velie E, Zhang Z. Incidence and survival of desmoplastic melanoma in the United States, 1992-2007. J Cutan Pathol. 2011;38(8):616-24.

13. Chen LL, Jaimes N, Barker CA, Busam KJ, Marghoob AA. Desmoplastic melanoma: a review. J Am Acad Dermatol. 2013;68(5):825-33.

14. Carlson JA, Dickersin GR, Sober AJ, Barnhill RL. Desmoplastic neurotropic melanoma. A clinicopathologic analysis of 28 cases. Cancer. 1995;75(2):478-94.

15. Debarbieux S, Ronger-Salve S, Dalle S, Balme B, Thomas L. Dermoscopy of desmoplastic melanoma: report of six cases. Br J Dermatol. 2008;159(2):360-3. 
16. Jaimes N, Chen L, Dusza SW, Carrera C, Puig S, Thomas L, et al. Clinical and dermoscopic characteristics of desmoplastic melanomas. JAMA Dermatol. 2013;149(4):413-21.

17. Hawkins WG, Busam KJ, Ben-Porat L, Panageas KS, Coit DG, Gyorki DE, et al. Desmoplastic melanoma: a pathologically and clinically distinct form of cutaneous melanoma. Ann Surg Oncol. 2005;12(3):207-13.

18. Palla B, Su A, Binder S, Dry S. SOX10 expression distinguishes desmoplastic melanoma from its histologic mimics. Am J Dermatopathol. 2013;35(5):576-81.

19. Tacha D, Qi W, Ra S, Bremer R, Yu C, Chu J, et al. A newly developed mouse monoclonal SOX10 antibody is a highly sensitive and specific marker for malignant melanoma, including spindle cell and desmoplastic melanomas. Arch Pathol Lab Med. 2015;139(4):530-6.

20. McKee P, Eduardo Calonje J. Diagnostic atlas of melanocytic pathology. 1st ed. New York: Mosby, Elsevier; 2009.

21. Cutler K, Chu P, Levin M, Wallack M, Don PC, Weinberg JM. Pedunculated malignant melanoma. Dermatol Surg. 2000;26(2):127-9.

22. Dini M, Quercioli F, Caldarella V, Gaetano M, Franchi A, Agostini T. Head and neck polypoid melanoma. J Craniofac Surg. 2012;23(1):e23-5.

23. Sidiropoulos M, Obregon R, Cooper C, Sholl LM, Guitart J, Gerami P. Primary dermal melanoma: a unique subtype of melanoma to be distinguished from cutaneous metastatic melanoma: a clinical, histologic, and gene expression-profiling study. J Am Acad Dermatol. 2014;71(6):1083-92.

24. Cabrera R, Pulgar C, Daza F, Castro A, Prieto VG, Benedetto J, et al. Dermatoscopy of a primary dermal melanoma. Am J Dermatopathol. 2009;31(6):574-7.

25. Feinsilber D, Kogan N, Rosati OM, Corbella C, Schröh R, Calb I. Verrucous melanoma: differences and similarities between primary and secondary varieties. Dermatol Argent. 2009;15(2):106-10.

26. Ohnishi T, Hamano M, Watanabe S. Clinically verrucous and histologically discohesive melanoma. A case report with dermoscopic and immunohistochemical observations. Australas J Dermatol. 2014;55(2):e21-3.

27. Chitu V, Zurac S, Cipi AE. Dermatoscopy of verrucous pigmented lesions is essential for choosing the appropriate treatment. Rom J Intern Med. 2015;53(4):359-64.

28. Coit DG, Thompson JA, Algazi A, Andtbacka R, Bichakjian CK, Carson WE 3rd, et al. NCCN Guidelines Insights: Melanoma, Version 3.2016. J Natl Compr Canc Netw. 2016;14(8):945-58.

29. Vyas R, Keller JJ, Honda K, Cooper KD, Gerstenblith MR. A systematic review and meta-analysis of animal-type melanoma. $\mathrm{J}$ Am Acad Dermatol. 2015;73(6):1031-9.

30. Aviles-Izquierdo JA, Leis-Dosil VM, Lazaro-Ochaita P. Animaltype melanoma: clinical and dermoscopic features of 3 cases. Actas Dermosifiliogr. 2014;105(2):186-90.

31. Seetharamu N, Ott PA, Pavlick AC. Mucosal melanomas: a casebased review of the literature. Oncologist. 2010;15(7):772-81.

32. Jethanamest D, Vila PM, Sikora AG, Morris LG. Predictors of survival in mucosal melanoma of the head and neck. Ann Surg Oncol. 2011;18(10):2748-56.

33. Lopez F, Rodrigo JP, Cardesa A, Triantafyllou A, Devaney KO, Mendenhall WM, et al. Update on primary head and neck mucosal melanoma. Head Neck. 2016;38(1):147-55.
34. Longo C, Piana S, Marghoob A, Cavicchini S, Rubegni P, Cota $\mathrm{C}$, et al. Morphological features of naevoid melanoma: results of a multicentre study of the International Dermoscopy Society. Br J Dermatol. 2015;172(4):961-7.

35. Stojkovic-Filipovic J, Kittler H. Dermatoscopy of amelanotic and hypomelanotic melanoma. J Dtsch Dermatol Ges. 2014;12(6):467-72.

36. Sbano P, Nami N, Grimaldi L, Rubegni P. True amelanotic melanoma: the great masquerader. J Plast Reconstr Aesthet Surg. 2010;63(3):e307-8.

37. Koch SE, Lange JR. Amelanotic melanoma: the great masquerader. J Am Acad Dermatol. 2000;42(5 Pt 1):731-4.

38. Chiaravalloti A, Banki A. An elusive amelanotic melanoma and review of dermoscopic findings. J Drugs Dermatol. 2017;16(11):1164-5.

39. Massi D, Pinzani P, Simi L, Salvianti F, De Giorgi V, Pizzichetta MA, et al. BRAF and KIT somatic mutations are present in amelanotic melanoma. Melanoma Res. 2013;23(5):414-9.

40. Hantschke M, Mentzel T, Kutzner H. Follicular malignant melanoma: a variant of melanoma to be distinguished from lentigo maligna melanoma. Am J Dermatopathol. 2004;26(5):359-63.

41. Tjarks BJ, Somani N, Piliang M, Bergfeld WF. A proposed classification for follicular involvement by melanoma. J Cutan Pathol. 2017;44(1):45-52.

42. McKinnon EL, West KL, Ball RA, Wright N, Barnhill RL. Folliculotropic Metastatic Melanoma: A Case Report and Review of the Literature. Am J Dermatopathol. 2017;39(9):e147-50.

43. Hu SW, Tahan SR, Kim CC. Follicular malignant melanoma: a case report of a metastatic variant and review of the literature. $\mathrm{J}$ Am Acad Dermatol. 2011;64(5):1007-10.

44. Machan S, El Shabrawi-Caelen L, Nikolay E, Kerl H, Requena L, Cerroni L. Follicular malignant melanoma: primary follicular or folliculotropic? Am J Dermatopathol. 2015;37(1):15-9.

45. Chapman I, Horton S, Liu W. Follicular malignant melanoma: a rare morphologic variant of melanoma. report of a case and review of the literature. Am J Dermatopathol. 2017;39(5):e69-70.

46. Banerjee SS, Eyden B. Divergent differentiation in malignant melanomas: a review. Histopathology. 2008;52(2):119-29.

47. Hayashi K, Okubo S, Watanabe T, Yamazaki Y, Horiuchi N, Saida T. Malignant melanoma on the sole showing prominent neural differentiation and perineural infiltration. Int J Dermatol. 2002;41(4):247-9.

48. Joana Devesa P, Labareda JM, Bartolo EA, Santos MF, Vale EM. Cartilaginous melanoma: case report and review of the literature. An Bras Dermatol. 2013;88(3):403-7.

49. Morimoto A, Asai J, Wakabayashi Y, Komori S, Hanada K, Takenaka $\mathrm{H}$, et al. Malignant melanoma with probable smooth muscle differentiation. Case Rep Dermatol. 2014;6(1):16-9.

50. Maher NG, Solinas A, Scolyer RA, Puig S, Pellacani G, Guitera P. Detection of desmoplastic melanoma with dermoscopy and reflectance confocal microscopy. J Eur Acad Dermatol Venereol. 2017;31(12):2016-24.

51. Pezzini C, Mandel VD, Persechino F, Ciardo S, Kaleci S, Chester J, et al. Seborrheic keratoses mimicking melanoma unveiled by in vivo reflectance confocal microscopy. Skin Res Technol. 2018;24(2):285-93. 